\title{
Numerical comparison between DPSK 33, DPSK 66 and duo binary in WOC system
}

\author{
Mustafa H. Ali ${ }^{1}$, Hazim M. Ali ${ }^{2}$, Tariq A. Hassan ${ }^{3}$ \\ ${ }^{1}$ Department of Basic Sciences, College of Dentistry, Mustansiriyah University, Baghdad, Iraq \\ ${ }^{2}$ General Directorate of Education Al-Najaf Al-Ashraf, Ministry of Education, Najaf, Iraq \\ ${ }^{3}$ Department of Computer science, College of Education, Mustansiriyah University, Baghdad, Iraq
}

\begin{tabular}{l} 
Article Info \\
\hline Article history: \\
Received Jul 16, 2020 \\
Revised Apr 29, 2021 \\
Accepted Jun 17, 2021
\end{tabular}

\section{Keywords:}

Differential phase shift keying modulation

Duo-binary

Inter satellite optical wireless communication

Modulation Format

\begin{abstract}
OWC systems have turned into a state-of-the-art technology because of their superior performance uniqueness and its innumerable characteristic as compared to its RF. One of the OWC application is inter-satellite wireless optical communication (Is-WOC), this framework can be improved by using different ways such as advanced modulation formats and aperture diameter. We demonstrate an analysis based on the aperture diameter effect in transmission of single channel with $40 \mathrm{~Gb} / \mathrm{s}$ Is-WOC system using three different modulation methods, differential phase-shift keying (DPSK) with a duty ratio to $66 \%$ and $33 \%$ and duobinary. Choosing these three modulations from various modulation format is done depending on the advantage they provide where the DPSK gives to fiber nonlinearity a powerful robustness and less susceptible to SPM and XPM and for system requirements of dispersion compensation in duobinary, it provides higher chromatic dispersion tolerance mitigate. The result shows the effect of different transmitter aperture diameter (from 2 to $20 \mathrm{~cm}$ ) for a constant distance 200 $\mathrm{km}$ accomplished by quality factor and minimum BER. We conclude there is a great loss in the small aperture diameter, even in the presence of best modulation format, therefore as the aperture diameter increase the Q-factor increase, but some increased rise is linear and the other is changing from linear to non-linear in a fixed Q-factor point equal to 5.63 .
\end{abstract}

This is an open access article under the CC BY-SA license.

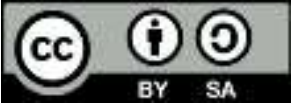

\section{Corresponding Author:}

Mustafa H. Ali

Department of Basic Sciences

College of Dentistry, Mustansiriyah University, Baghdad, Iraq

Email: mustafa.h@uomustansiriyah.edu.iq

\section{INTRODUCTION}

The first theoretical study was done by Fried in 1967, and after that various theoretical studies were suggested. in the mid-1980s and as a result of many research in Is-OWC link, the european space agency (ESA) start a new project called semiconductor inter-satellite laser experiment (SILEX) program to develop the optical communication between various satellite. Between 1990 and 1992 Some of the earlier ground-tosatellite communication experiments include uplink based RME and GOPEX. After that a two-way communication link GOLD starts to develop in 1995 [1]. The first inter satellite link (ISL) was started in the year 2001 between ARTEMIS and SPOT-4 by ESA French Earth surveillance in a condition that has minimum earth orbit and sun synchronous with 50 Mbps data capacity. Later a link between a KIRARI and ARTEMIS was successfully built in 2005. In 2008, Terra SAR-X and NFIRE were linked using optical communication. Several other experiments have been performed till now in the area of Is-OWC systems and research are still going on to improve system performances [2]-[4]. 
The rapidly increasing to bandwidth claiming in access networks is because of the huge popularity of various services in multimedia like high-speed online gaming, high-definition television (HDTV), voice and video-over-internet protocol (VoIP) and mass-data [5]. This expands put burden on communication limited, in 2009, there were almost near 6124 satellites hovering around the globe and this number has raised depending on the radio frequency (RF) raised spectrum [6]. Beside RF suffer from limited in terms of low data rates, less capacity, more interferences, crowded spectrum, higher installation costs and very expensive licensing, RF rays have been exploited in a number of ways and now there is a strong need to find a suitable replacement in possible areas [7]-[9].

As shown in Figure 1, a state-of-the-art technique is floating on the scene to substitute the utilize of microwave or RF technique for transmission of signals and data [10], [11]. This new technique called free space optical communication (FSO) [12]. When FSO uses in Inter Satellite links it called IS-OWC. It is distinguishing over FSO due to it is main job is to connect two satellites with optimum performance using optical communication propagation, it means IS-OWC are free from atmospheric instabilities like snow, rain, dust, fog, mist and scintillation effects that the FSO has [13]. IS-OWC systems as obvious from its name is used as an optical communication among two satellites for transport, optical signal over guided wireless links driving to an incorporation of wireless and optical transition [14], [15].

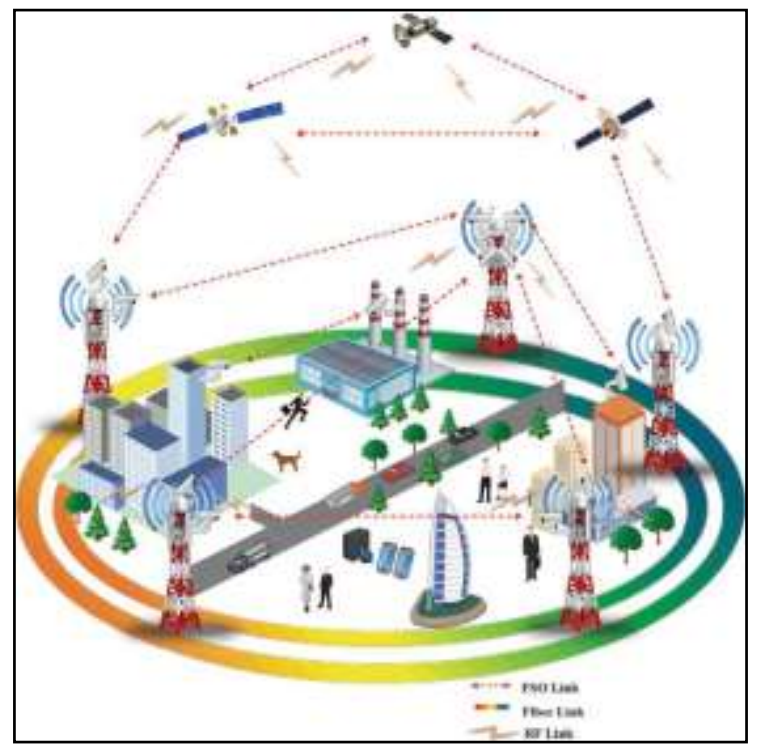

Figure 1. FSO, fiber optic and RF running our worldwide communication [10]

In another word this system has built up a landmark and turn into a hopeful architecture resolution for next generation access networks. After this success, it will conquer the communication world in enormous implementation, that it is extremely complicated to diffuse optical fiber cables for long distance and contains certain merits over the current microwave communication. Is-OWC domination came from ability of improvement lasers that capable to send data up to some thousands of kilometers, low cost, high speed, small size of the antenna, less bit-errors, excellent bit-rate, low power consumption, long distance transmission applications, high security, no EMI interference, no license is required, simple maintenance, easy operation, longer lifetimes and high bandwidth provision point to point communication [16]-[20].

Even with the privilege mention before in Is-OWC both satellite link and ground station are residue in microwave method, but in space the link connecting two satellites is substituted by optical signal. Is-OWC spectrum available for optical system is unrestricted and it is 10,000 times more than RF rays due to the very high frequency range that can operate with it, in addition, it has the capability to work in various electromagnetic bands such as infrared wavelength (750-1560 NM) and ultraviolet communication (200-280 NM) [21]. At last, it reduces the area of the payload and weight of satellites because components required are compact and light in weight as compared to RF based satellite communication as shown in Figure 2.

With the increase in capacity of transmission system, interest in modulation formats with high spectral efficiency is developed. Therefore, superior modulation code is desired. On the other hand, having a 
narrow optical spectrum led to not only enhance the spectral efficiency but also resist more distortion. Consequently, selecting modulation format shows a crucial role in the time we design any network for communication proficiently. This work provides a relative analysis, among some highly used advanced modulation formats such as, DB and differential phase-shift keying (DPSK) 33, DPSK 66 for a single channel transmission link operating at a bit rate of $40 \mathrm{~GB} / \mathrm{s}$ and $200 \mathrm{~km}$ distance. Characterization of modulation methods has been explained based on Q factor and Eye opening, consequently, results have been analysis with future scope and conclusion to decide the effectiveness of IS-OWC system under these circumstances represent by different transmitter aperture diameter.

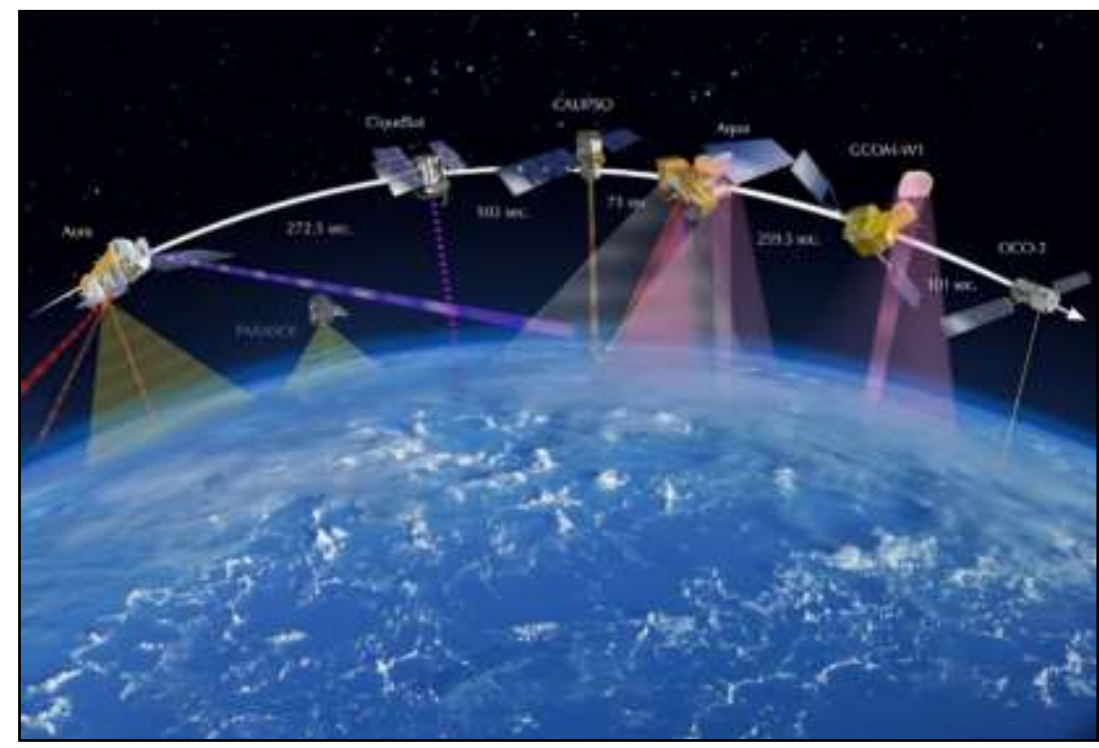

Figure 2. Different satellite hovering around the globe [20]

In introduction side we demonstrate the important of OWC systems and how it turned into a stateof-the-art technology, In the material and method side We demonstrate an analysis based on the Aperture Diameter effect in transmission of single channel with $40 \mathrm{~Gb} / \mathrm{s}$ Is-WOC system using three different modulation methods, differential phase-shift keying (DPSK) with a duty ratio to $66 \%$ and $33 \%$ and duobinary. The result side shows the effect of different Transmitter aperture diameter (from 2 to $20 \mathrm{~cm}$ ) for a constant distance $200 \mathrm{~km}$ accomplished by quality factor and minimum BER. Then the conclusion shows there is a great loss in the small aperture diameter, even in the presence of best modulation format.

\section{RELATED WORK}

A. Kaur and A. Kaur Brar [21] a mode division multiplexed inter-satellite optical wireless communication is presented at different data rates such as $10 \mathrm{Gbps}, 20 \mathrm{Gbps}$ and $40 \mathrm{Gbps}$ in terms of Q factor. Presented system is accentuated towards the performance evaluation of MD-DQPSK (modified differential phase shift keying) modulation at different distances $(750 \mathrm{~km}$ to $3750 \mathrm{~km}$ ). Linear polarized (LP) modes, 64 in number are incorporated in the system to carry the high-speed data. Further performance of proposed MD-DQPSK-MDM Is-OWC is compared with differential quadrature phase shift keying (DQPSK), Manchester coding and DPSK (differential phase shift keying). Q factor of MDRZ- DQPSK, DPSK, DQPSK and Manchester coding at $2500 \mathrm{~km}$ of link distance are observed as 16, 8.4, 10.37, and 3.56 respectively at 40 Gbps. Results revealed MD-DQPSK provide highest Q factor in the contrast to DQPSK, DPSK and Manchester modulation.

N. Badhan and N. Sharma [22] in this work, comparative analysis of a single channel 40Gb/s system is done using duo-binary, differential phase shift keying and carrier suppressed return-to-zero modulation formats. Performance is analyzed for the factors (different compensating schemes, number of spans and amplified spontaneous emission noise), with four number of spans, each span equal to $50 \mathrm{~km}$. The result for all three formats shows the Effects of ASE at the end of 4th span and differentiation is done by monitoring the spectrum of optical signal noise. 
P. Kaur et al. [23], This paper looks into the achievement of DWDM multiplexing technique in IS-OWC system link using NRZ and RZ modulation formats for long distance transmission. The system has been analysis by varying the levels of input power to transmit $10 \mathrm{Gbps}$ for communication up to 5000 kilometers. H. Kaur et al. [24], the article displays an inclusive method of designing MDM in IS-OWC system utilizing MDRZ-DQPSK, CSRZ-DQPSK and DRZ-DQPSK modulation system. The system has 64 channels with various data rates and varied distance from 900 to $4500 \mathrm{~km}$ at the result shows at $10 \mathrm{Gbps}$ bit rate and distance up to $4500 \mathrm{~km}$ the MDRZ-DQPSK modulation gives an excellent Q-factor compared to DRZ-DQPSK and CSRZDQPSK Modulation, however due to high consumption of bandwidth in data rate 40 Gbps CSRZ-DQPSK exhibits least Q-factor therefore, it preferable for long reach transmission and saves cost.

P. Kaur and B Utreja [25], in this work present a comparison between different modulation formats DRZ, CSRZ and DPSK in IS-OWC links. The results show that DPSK that has less cost and simple design at the data rate of $40 \mathrm{Gbps}$ performs better than the other modulation format in higher transmission range. We notice that this significant advance in the achievement of DPSK system is duo to the increasing aperture diameters of both receivers and transmitters. While DRZ and CSRZ perform better for small distance.

H. Kaur and H. Kaur [26] in this work the 64 channels in DWDM system with 40 Gbps data rate is design to show it is best performance with DPSK modulation system. The result shows that DPSK give optimum efficiency when work in DWDM systems. However, the nonlinearities affect must be taken under consideration are in the time developing such system.

The first part of the paper was the introduction that divided to (historical background, the problem, the proposed solution and related work), the second part to talk about the properties of modulation modulation type (DPSK 33, DPSK66 and DB) characterization and simulation, the tools and that we need to design and build an integrated pocket system. Then, in the third part, the results that appeared in the design of the system were demonstrated under the influence of variables aperture diameter, and finally in the fourth part show our analysis conclusions from these variables.

\section{MODULATION METHODS CHARACTERIZATION AND SIMULATION}

The simulation of a $40 \mathrm{~GB} / \mathrm{s}$ single channel transmission link using a different modulation formats signal is fed to IS-OWC channel was done using Optisystem program 7.0 that has the ability to simulate a various equipment and connecting them in multiple design to provide an efficient system with minimum error and low cost. Length of OWC channel is fixed at $200 \mathrm{~km}$. We ideally assumed the additional losses because of atmospheric effects and zero attention. The OWC channel represents the medium among receiver and transmitter. For Is-OWC system, Line-of-Sight is required among receiver and transmitter. The aperture diameter of transmitting and receiving antenna represent a remarkable part in such systems where the mode of transmission is wireless. The major advantage of using light as carrier for information transfer is that antennas with small aperture diameters are required which leads to light weight and easily manageable systems, Therefore, we used aperture antenna diameter from $2 \mathrm{~cm}$ to $20 \mathrm{~cm}$ in the receiver and transmitter side. There is no divergence because there is no air in wireless, optical communication based on inter-satellite signal transmission. We do not use any power amplifiers before or after the $200 \mathrm{~km}$ OWC channel for the compensation of the amplitude degradation effects so it became all passive.

We chose a wavelength in IR-B (1400-3000nm) band in the region of short infrared (1530-1560 nm) duo to low attenuation characteristics in this wavelength section, specialty 1552.52 due to low power consumption and no need to a mechanical part associated with IR-B connection. 1552.52 is most exploited wavelengths as long the wavelength laser will be the bad impacts of Rayleigh and Mie scattering also it is compatible with existing equipment's (1):

$$
P_{r}=P_{T} \eta_{T} \eta_{R}\left(\frac{\lambda}{4 \pi \mathrm{Z}}\right)^{2} G_{T} L_{T} G_{R} L_{R}
$$

where $\mathrm{Z}$ is the distance among receiver side and transmission side, $\lambda$ is wavelength, $\eta_{R}$ is the efficiency of optical receiver, $\eta_{T}$ is efficiency of optical transmitter, $P_{T}$ is the transmitter optical power. $G_{R}$ receiver gain are given by (2), $\mathrm{G}_{\mathrm{T}}$ are transmitter gain given by (3);

$$
\begin{aligned}
& G_{R}=\left(\frac{\pi D_{R}}{\lambda}\right)^{2} \\
& G_{T}=\left(\frac{\pi D_{T}}{\lambda}\right)^{2}
\end{aligned}
$$

Indonesian J Elec Eng \& Comp Sci, Vol. 23, No. 1, July 2021: 247 - 257 
$\mathrm{L}_{\mathrm{R}}$ is the reception pointing loss factor are given by (4). $\mathrm{L}_{\mathrm{T}}$ are transmission pointing loss factor are given by (5),

$$
\begin{aligned}
& L_{R}=\exp \left(\frac{-G_{R}}{\theta_{R}{ }^{2}}\right) \\
& L_{T}=\exp \left(\frac{-G_{T}}{\theta_{T}{ }^{2}}\right)
\end{aligned}
$$

keeping the value of $\mathrm{Z}$ to be $200 \mathrm{k}$ in the designed system will decrease the losses. Where $\theta$ is the full transmitting divergence angle, $\mathrm{D}$ is the receiver diameter, as shown in equation $7 \mathrm{P}_{\mathrm{R}}$ usually contains $\mathrm{P}_{\mathrm{T}}$

$$
P_{T}=P_{R} \exp \left(-T_{o d}\right)
$$

$\mathrm{T}$ is the Beam transmit power and can be explain by (7):

$$
T=P_{R} / P_{T}=P_{R} \exp \left(-T_{o d}\right)=\exp (-\alpha(\lambda) L)
$$

where $\mathrm{L}$ is the length of transmission taken in $\mathrm{m}, \mathrm{T}_{\mathrm{od}}$ is the depth of optical beam, and $\alpha$ is the attenuation taken in $(1 / \mathrm{m})$.

Filter selection: The purpose of utilizing an electronic filter in Is-OWC system is to accept required signal and reject all other noises. Low Pass Bessel filter with cutoff frequency $0.75^{*}$ Bit rate is the most commonly used filters in Is-OWC. The effect of using different filters on system efficiency in terms of Q-factor and BER, Results from different studies, like a show that Bessel, Cosine Roll off and Gaussian filters are best for Is-OWC.

The simulation study of in IS-OWC channel with three modulation code (DPSK 66, DPSK 33 and the DB) has been analyzed, the whole system is split up into three parts main components: Transmitter or satellite 1 , transmission channel and receiving transponder or satellite 2 .

\subsection{Differential, phase shift keying modulation (DPSK)}

Differential phase-shift keying (DPSK) is one the most promising modulation formats in phase shift keyed. In DPSK modulation format, bits are again represented in terms of shift in phase. Bit 1 corresponds to 180 phase shift and bit 0 represents no shift in phase. The benefit of this method is the high spectral effectiveness as it predictable to supply a resolution for achievement $40-\mathrm{Gbps}$. For every single channel with a bit rate is the $40-\mathrm{Gb} / \mathrm{s}$. Duo to diverse in the channel count from to 33-each and 66-ch, therefore we take both these two duty cycles, the main advantages of DPSK:

- The DPSK modulation format has confirmed their flexibility to nonlinearities, higher tolerance to chromatic dispersion (up to a certain limit), can deal with higher bit rate, high tolerance to doppler-shift, less complications in tracking and longer transmission range. The fixed density nature of the DPSK minimizes the effect of fiber nonlinearity pending transmission. Thais improve the system power budget and make it more effective than QPSK and 8-DPSK as mentioned above.

- DPSK Preference over similar systems like amplitude shift keying (ASK) because DPSK system incompatible with a 3-dB higher sensitivity, balanced detection, and is more durable on filtering narrowband optical signal.

- DPSK superior receiver sensitivity, less problems in tracking and perfect tolerance to signal degradation that raised by transmission fiber nonlinearity.

- Newly, it has been explained from a nonlinear loop and the balance between semiconductor optical amplifier and delayed interferometer combination, that QPSK signal quality could be enhanced.

As shown in our schematic setup Figures 3 and 4, the transmitter consists of pseudo-random bit sequence (PRBS) generator which supply the signal to the fork with $1 * 2$ port, his job is to divide the signals to NRZ pulse generator and binary NOT (in case of DPSK 33) than to duobinary procedure. In case of DPSK 66 the fork divides the signals to NRZ pulse generator and duobinary procedure on the first output of the fork, as we mention there is The NRZ signal which used to generate the final BER analyzer, on the second output of the fork represent the main course of the system which feed the Li-Nb MZ modulator by 3 inputs, one from the continuous wave laser, and the other two inputs are the phase modulation that generated by encoding the NRZ signal utilizing a duobinary procedure.

With the frequency of $20 \mathrm{GHz}$, the signal taken from the output of $\mathrm{Li}-\mathrm{Nb} \mathrm{MZ}$ modulator is inter into the sinusoidal electrical signal and then concatenating to a fork and electrical gain. The driven signal from the second modulator is generated depending on the input first direct signal from the electrical sinusoidal, second from the fork and third from the fork after inter into electrical gain. 


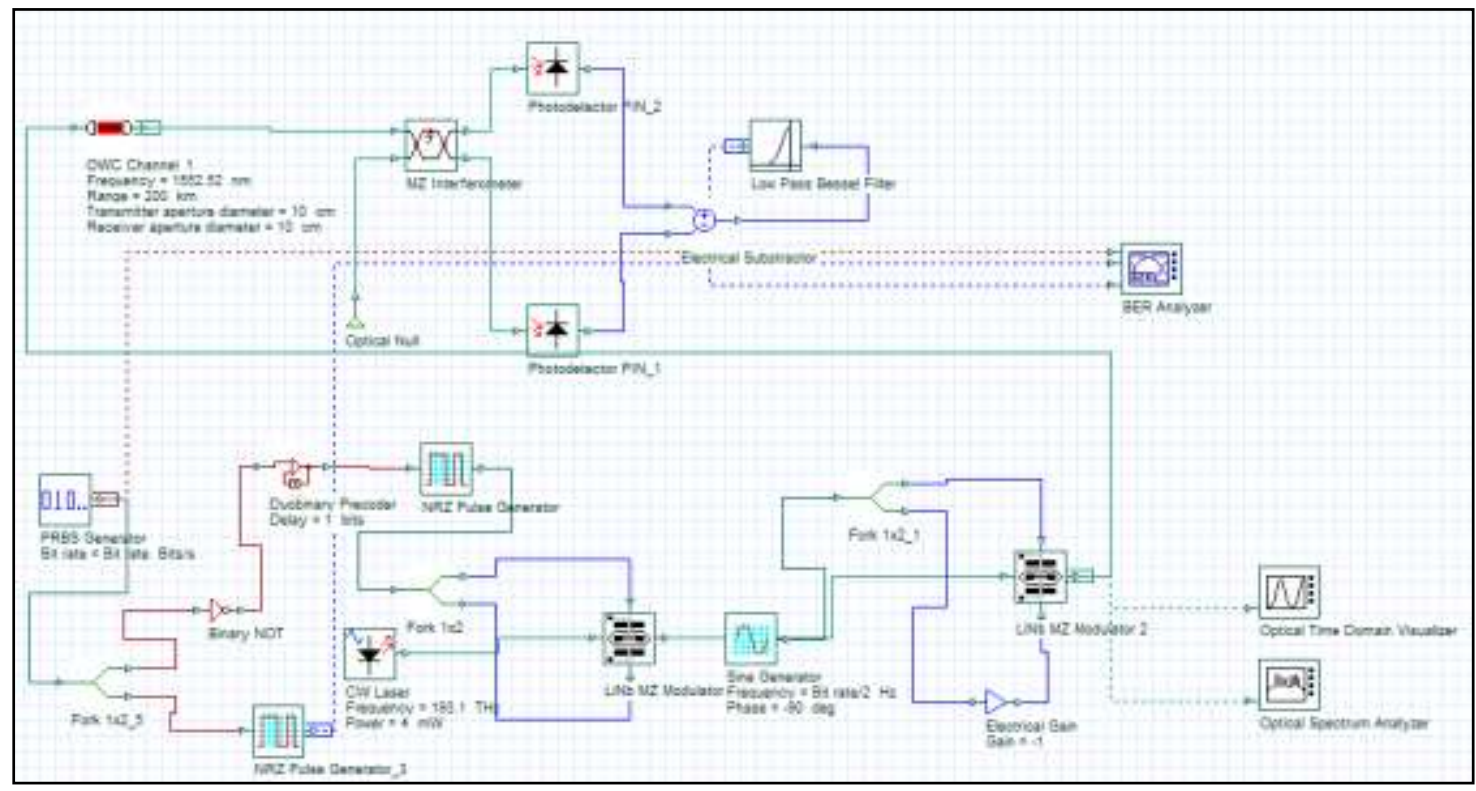

Figure 3. Internal simulation structure of DPSK 33

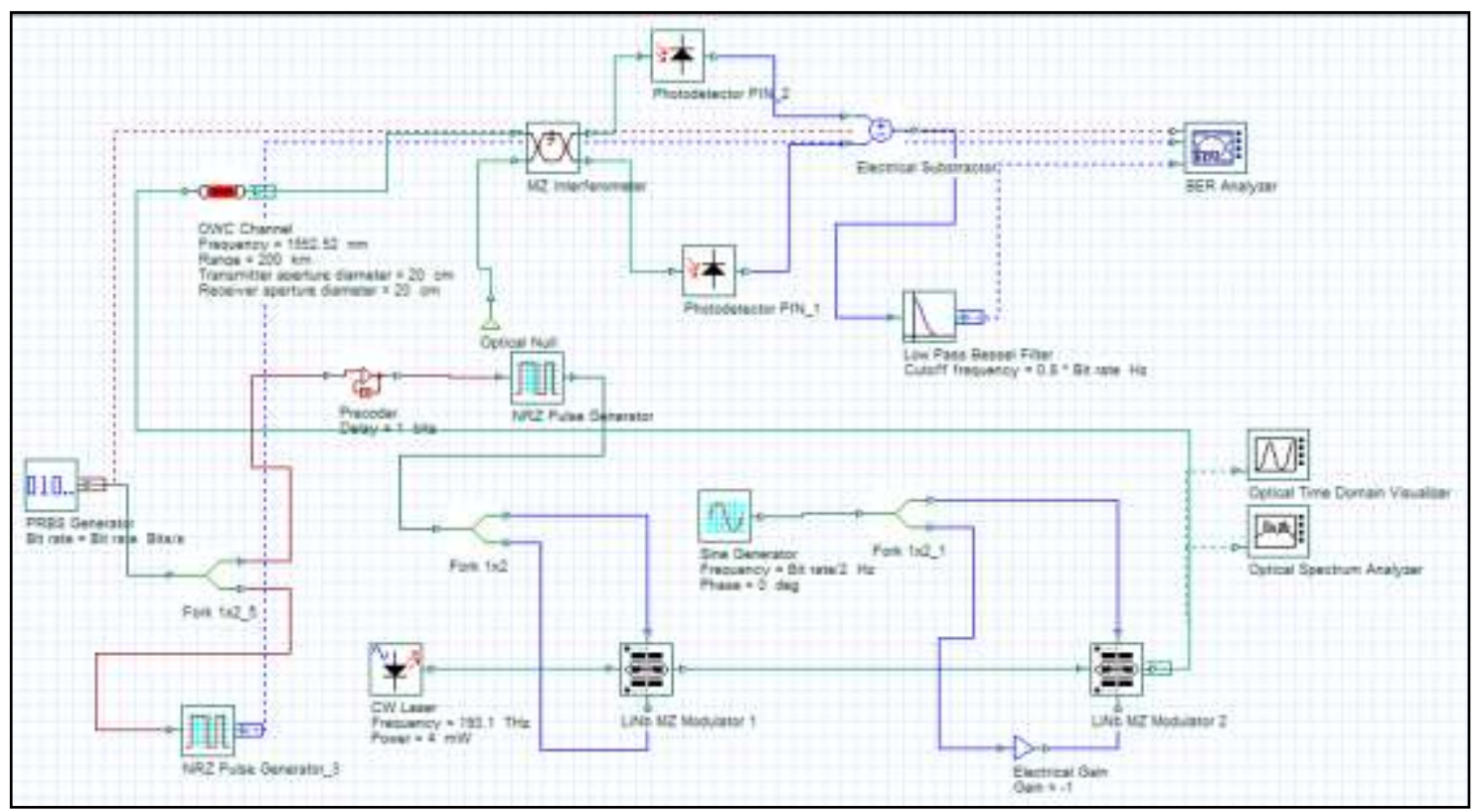

Figure 4. Internal simulation structure of DPSK 66

\subsection{DPSK 66}

Now we have our signal readiness to transmit through OWC. We should note that, the phase of bits ' 1 's is alternating with a variation of 180 no. This phase variation is the reason of eliminating the $193.1 \mathrm{THz}$ carrier. On the receiver side, we need a transducer that can convert the optical signal to an electrical signal, therefore we use a Mach-Zehnder interferometer and balanced photodiodes, to demodulate the transmitted signal and generate the encoding initial bit sequence. Then combine the two balanced photodiodes output signal into electrical subtractor which finally to low pass bessel filter to extract the original signal from the carrier.

BER analyzer allow as to draw a graph between Q factor and time period. We can see from the design above there is almost no change from DPSK 33, all simulations work the same except the small differ electrical sinusoidal phase in DPSK 66 equal to 0 in the state of -90 in DPSK 33 and there is no binary not before duobinary precoder in DPSK 66. 


\subsection{Duo-binary (DB)}

The duo-binary (DB )transmission is functional for 40-Gb/s as well-known, light pulse having phase of 0 or 180 represents bit 1 and bit zero is represented by the absence of light. The phase of bit 1 is selected in a controlled way that there will $\pi$ phase shift between bit 1 separated by bits containing an odd number of 0 's.

- The duobinary modulation format (DBM) is more common when it compares with other type of modulation due to dose not use any kind of repeaters in spite that can cover the long transmission distance.

- With direct detection the DB signals are a cost-effective method for 40 Gbps technology.

- DB is an attractive modulation format which has a great tolerance to the effect of low spectral tenancy and chromatic dispersion through bandwidth reduction and its narrow spectral width this make it proficient for high spectral effectiveness, high data transmission rate and significant in fiber systems with standard requirements, therefore it is dispersion limited in the system performance.

- As the bandwidth demand for broadband networks and high-speed systems has raised. DB signaling has turned into a considerable transmission format, therefore choosing optical modulation format through selecting appropriate pulse shaping has turned into a substantial standard in any design devoted for high speed.

- The essential idea of DB is to deliberately encourage the USE by interfering data from neighboring bits. This is done by adding a data series to a 1-bit delayed version of itself.

DB pulse forms for are explain in the below (8).

$$
X(t)=\sin c\left(\frac{t}{T}\right)+\sin c\left(\frac{t-T}{T}\right)
$$

As shown in Figure 5, using a NRZ pulse generator and duo-binary procedure for generating duobinary pulses then after pass in electrical delay to shape these pulses and enter into Mach-Zehnder modulator (MZM) bias points. After that, these pulses are interring to MZM in the electrical gain for the two modulators as shown in the figure. For duobinary pulses direct detection we using the PIN photo detector to detect the DB pulses, choosing PIN are coming from it is simplicity. Finally, to achieve better optical receiver sensitivity, MZM should be biased at the null point because of lower noise equivalent bandwidth. There are two reasons behind using DPSK modulation format first one it has fixed optical power consequently less sensitive to XPM and SPM but not against chromatic dispersions. Another reason is the improvement in 3-dB receiver sensitivity in the contrast to NRZ. DPSK has a higher BER value that is equal to $5.26 * 10-8$ at $0 \mathrm{dBm}$ and $4.94 * 10-324$ at $30 \mathrm{dBm}$. It means as power level is raised BER began to decreasing but for DPSK it remains the same.

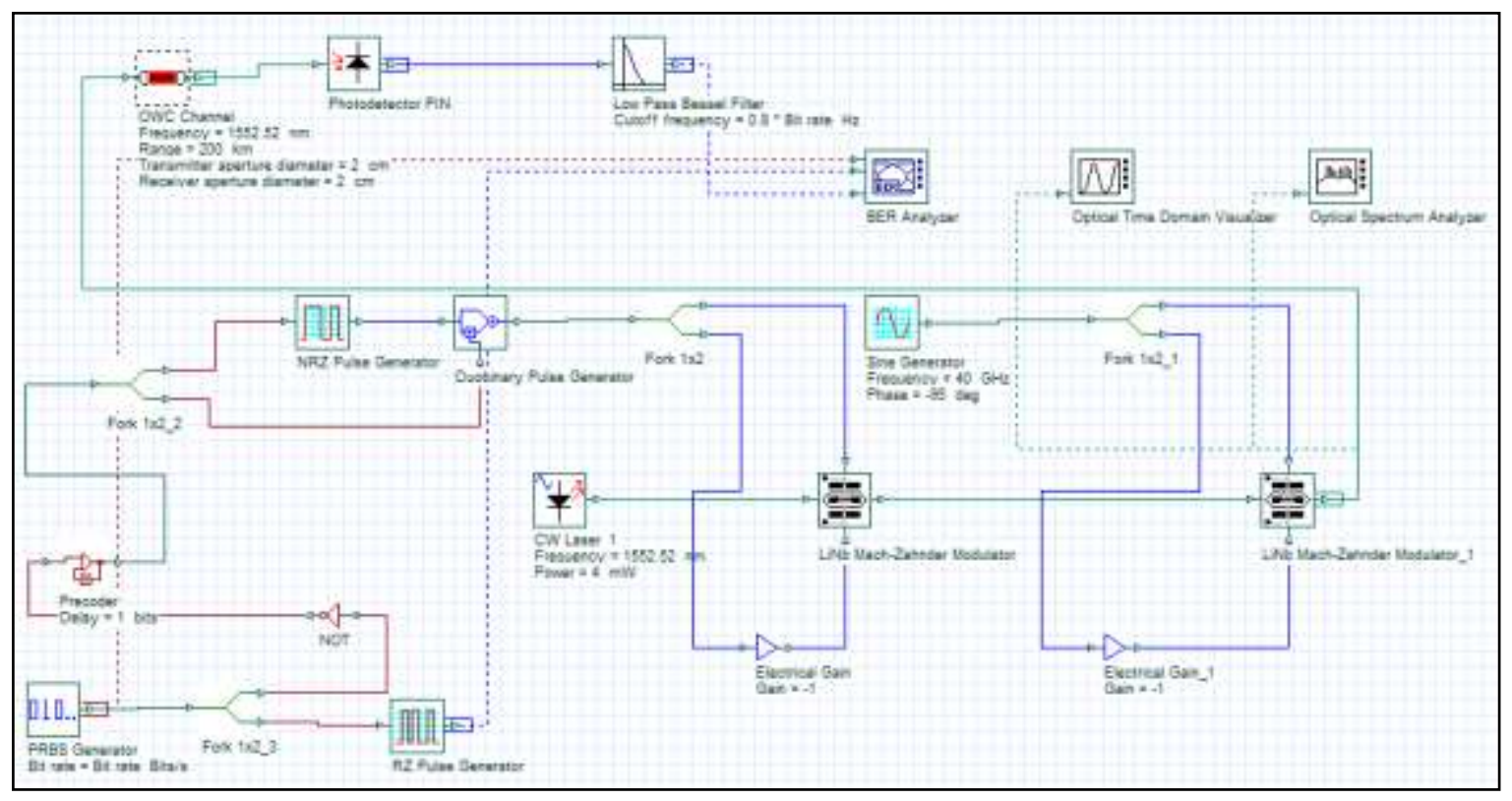

Figure 5. Internal simulation structure of DPSK 66 


\section{RESULT AND DISCUSSION}

Three modulation formats are analyzed using A single channel optical wireless with results are evaluated in terms of eye opening and Q factor, in our design with a constant distances $200 \mathrm{~km}$ in LEO orbit, wavelength equal to $193.1 \mathrm{THz}$ and $4 \mathrm{~mW}$ input power. The various transmitter aperture diameters from $2 \mathrm{~cm}$ to $20 \mathrm{~cm}$ gave us steady performance in all receiver aperture diameters in case of DPSK 66 which is the best compared to the other type of modulation format on the other hand, as shown in Figures 6-15, the DB and DPSK 33 are hard to say which one is better than the other because when we increase the transmitter aperture dimeter more than $4 \mathrm{~cm}$, we notice the q factor of DPSK 33 is shows better until a certain point of Q-factor equal to 5.63 it reverses and the DB is become the better $q$ factor.

In Figure 16, the greater eye-opening factor indicates better signal quality and the more eye closes, it is hard to differentiate between bit one and zero in the signal. DPSK 66 shows steady performance the eye-opening factor of DPSK66 much bigger then, DB and DPSK 33. In this point 5.63 both the two coding formats is becoming equal, neglect the receiver and transmitter aperture diameter except the case of Figure 6 with $2 \mathrm{~cm}$ transmitter aperture diameters that show DPSK 33 is better than DB in all receiver aperture diameters. Below this point the DPSK 33 is better in performance than DB, above this point the DB is better than DPSK.

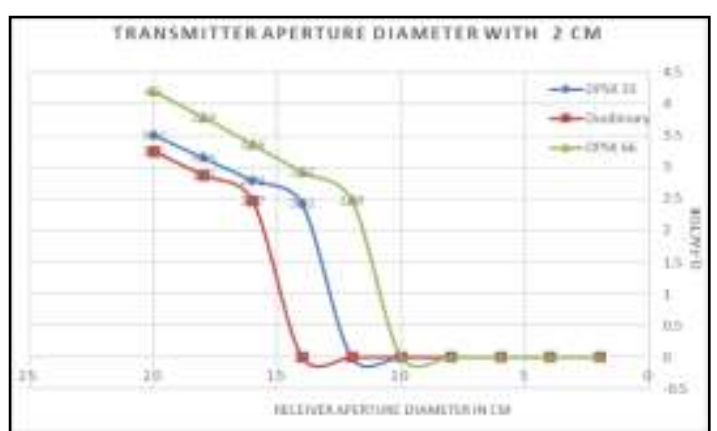

Figure 6. Transmitter aperture diameter with $2 \mathrm{~cm}$

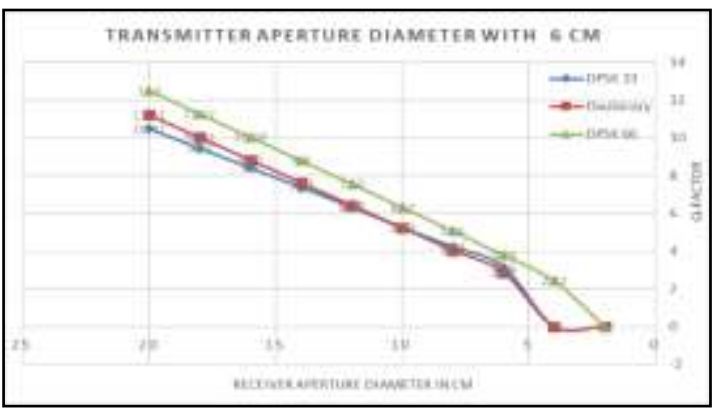

Figure 8. Transmitter aperture diameter with $6 \mathrm{~cm}$

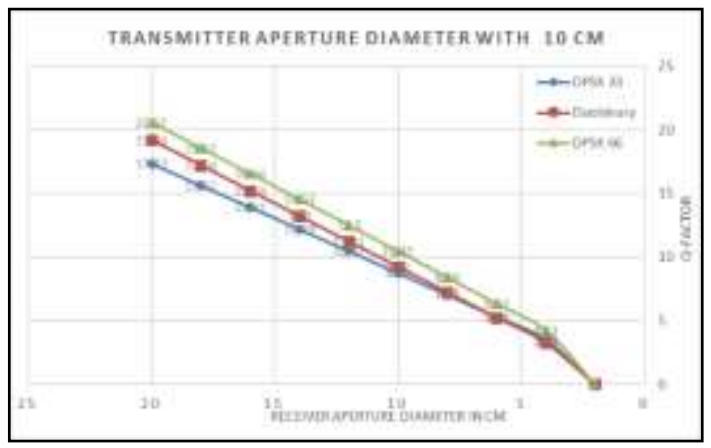

Figure 10. Transmitter aperture diameter with $10 \mathrm{~cm}$

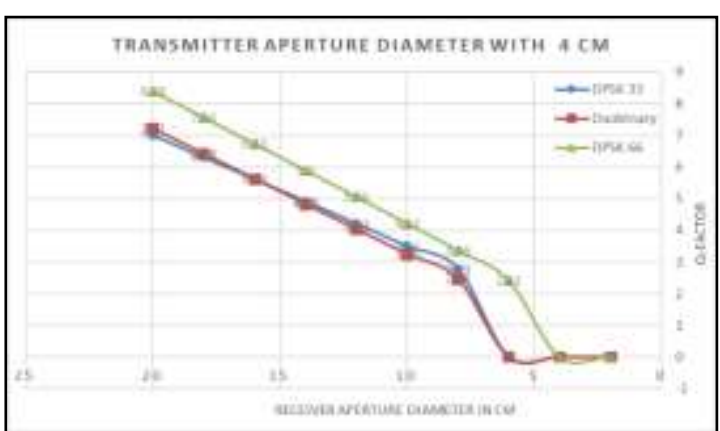

Figure 7. Transmitter aperture diameter with $4 \mathrm{~cm}$

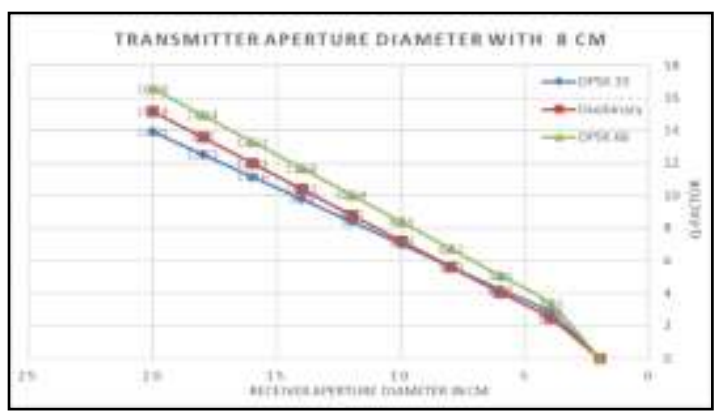

Figure 9. Transmitter aperture diameter with $8 \mathrm{~cm}$

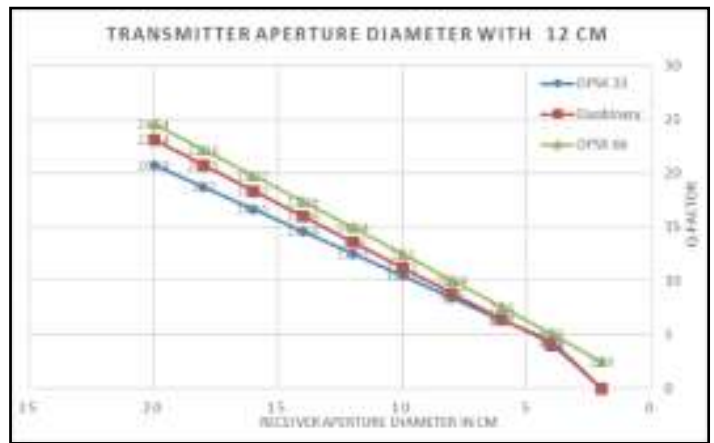

Figure 11. Transmitter aperture diameter with $12 \mathrm{~cm}$ 


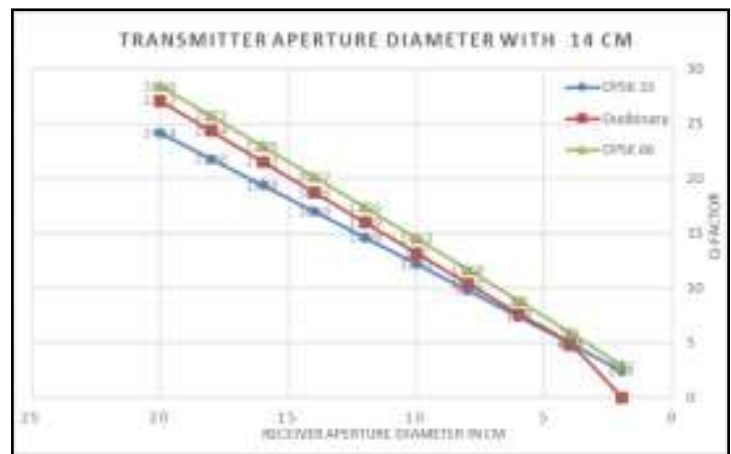

Figure 12. Transmitter aperture diameter with $14 \mathrm{~cm}$

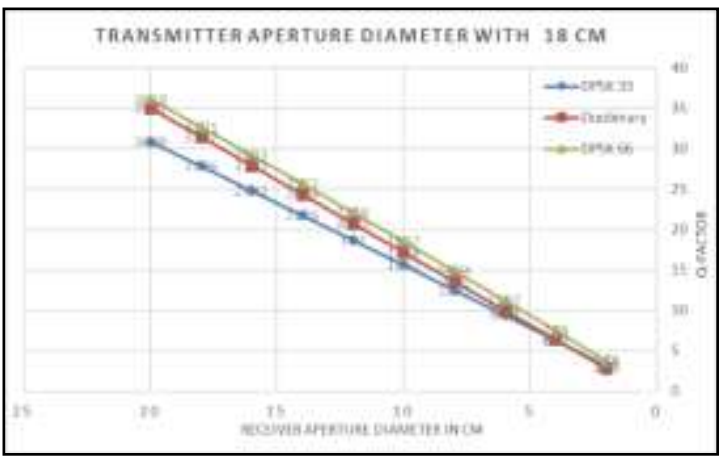

Figure 14. Transmitter aperture diameter with $18 \mathrm{~cm}$

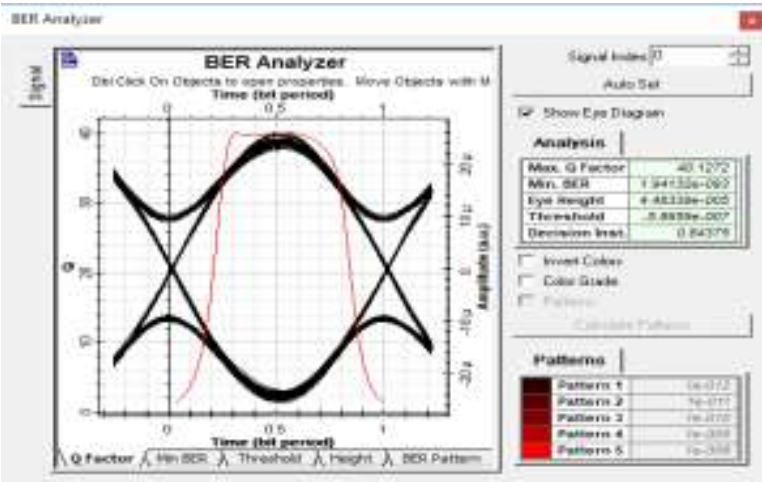

(a)

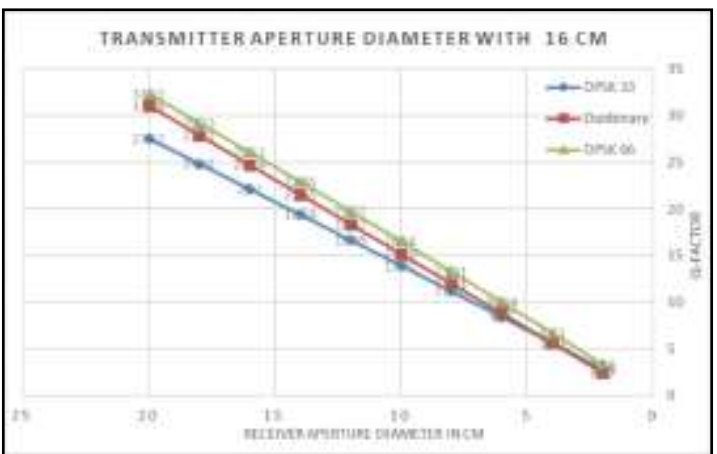

Figure 13. Transmitter aperture diameter with $16 \mathrm{~cm}$

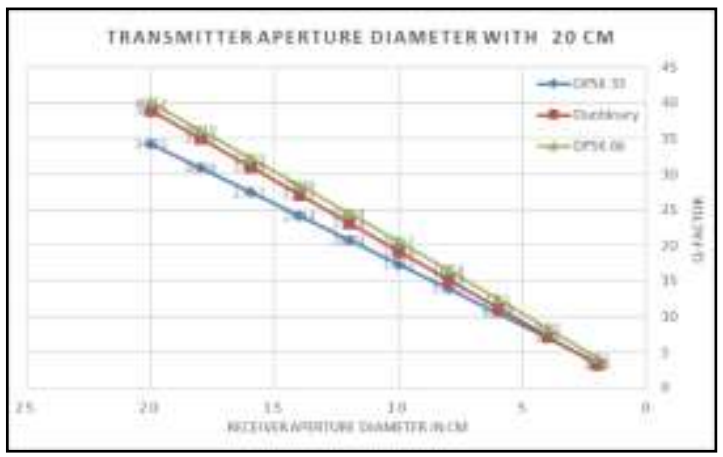

Figure 15. Transmitter aperture diameter with $20 \mathrm{~cm}$

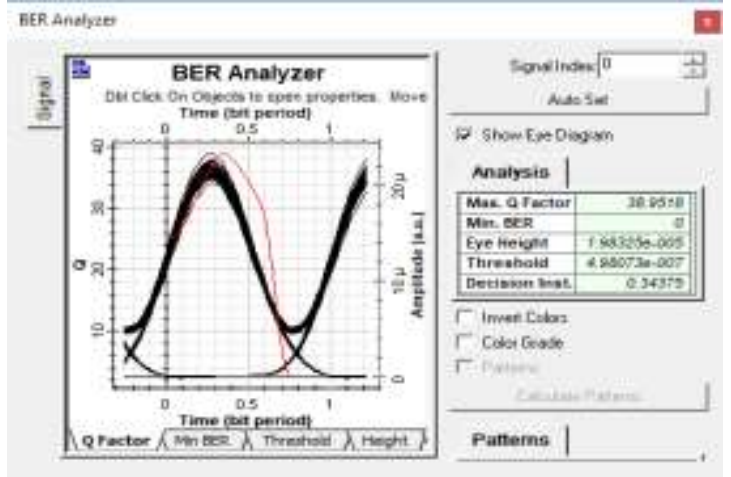

(b)

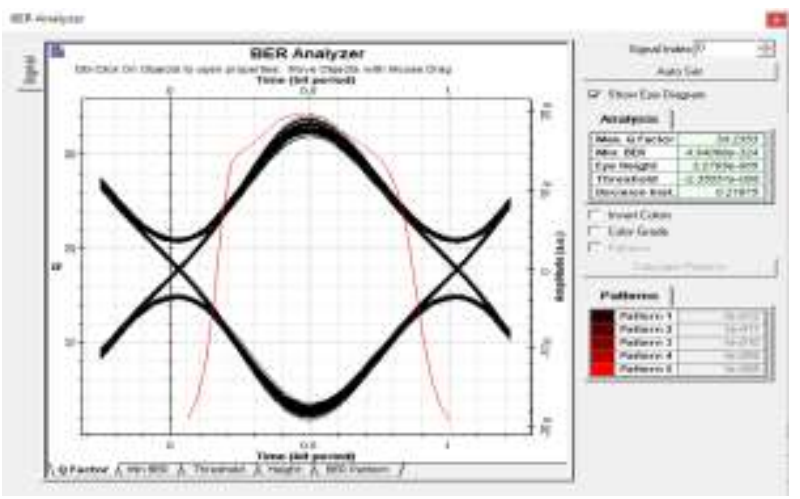

(c)

Figure 16. Eye opening factor of; (a) DPSK66, (b) DB and, (c) DPSK 33 


\section{CONCLUSION}

At constant data rates equal to $40 \mathrm{Gbps}$ an improvment of the capacity in Is-OWC system is done by comparative analysis of various modulation format. It shows that the best performance basis on the minimum BER and Q-factor has been accomplished by DPSK 66\% which achieve the best result in all the aperture diameter in the contrast to DPSK 33 and duobinary modulation techniques. On the other hand, it is also observed that the difference in the behavior of DPSK 33 and doubinary in their Q-factor is that there is non-linear shape as the aperture diameter increase. Starting from aperture diameter $2 \mathrm{~cm}$ both q-factor and eye opening behaves is linear (for both DPSK 33 and doubinary), but as the transmitter aperture diameter increase the Q-factor of the DPSK 33 is continue to raise linearly, unlike the doubinary which start smaller than the DPSK 33, unlit it reaches some point of Q-factor equal to 5.63 then the it began to increase faster than DPSK 33. These value 5.63 is fixed for all aperture diameters where the DPSK 33 and doubinary are equals.

\section{ACKNOWLEDGEMENTS}

The authors would like to thank Mustansiriyah University (www.uomustansiriyah.edu.iq) Baghdad, Iraq for it is support in the present work.

\section{REFERENCES}

[1] A. García-Zambrana, C. Castillo-Vázquez and B. Castillo-Vázquez, "Rate-adaptive free-space optical links over atmospheric turbulence and misalignment fading channels," Optical Communication, Intech open book chapter, pp. 321-340, 2012, doi: 0.5772/45783.

[2] A. R. Darlis, L. Jambola, L. Lidyawati and A. H. Asri, "Optical repeater for indoor visible light communication using amplify-forward method," Indonesian Journal of Electrical Engineering and Computer Science, vol. 20, no. 3, pp. 1351-1360, December 2020, doi: 10.11591/ijeecs.v20.i3.pp1351-1360.

[3] A. Ramezani, M. R. Noroozi and M. Aghababaee, "Analyzing free space optical communication performance," International Journal of Engineering and Advanced Technology (IJEAT), vol. 4, no. 1, pp. 46-51, 2014,

[4] M.S. M. Gismalla and M.F. L. Abdullah, "Performance evaluation of optical attocells configuration in an indoor visible light communication," Indonesian Journal of Electrical Engineering and Computer Science, vol 14, no. 2, pp. 668-676, May 2019, doi: 10.11591/ijeecs.v14.i2.pp668-676.

[5] M. Ijaz, Z. Ghassemlooy, J. Pesek, O. Fiser, H. Le Minh and E. Bentley, "Modeling of Fog and Smoke Attenuation in Free Space Optical Communications Link Under Controlled Laboratory Conditions," in Journal of Lightwave Technology, vol. 31, no. 11, pp. 1720-1726, June1, 2013, doi: 10.1109/JLT.2013.2257683.

[6] S. A. Al-Gailani, A. B. Mohammad and R. Q. Shaddad, "Evaluation of a $1 \mathrm{~Gb} / \mathrm{s}$ Free Space Optic system in typical Malaysian weather," 2012 IEEE 3rd International Conference on Photonics, 2012, pp. 121-124, doi: 10.1109/ICP.2012.6379839.

[7] Y. Li, M. Pióro and V. Angelakisi, "Design of cellular backhaul topology using the FSO technology," 2013 2nd International Workshop on Optical Wireless Communications (IWOW), 2013, pp. 6-10, doi: 10.1109/IWOW.2013.6777766.

[8] F. Nadeem, B. Flecker, E. Leitgeb, M. S. Khan, M. S. Awan and T. Javornik, "Comparing the fog effects on hybrid network using optical wireless and GHz links," 2008 6th International Symposium on Communication Systems, Networks and Digital Signal Processing, 2008, pp. 278-282, doi: 10.1109/CSNDSP.2008.4610745.

[9] R. Lange and B. Smutny, "Homodyne BPSK based optical inter-satellite communication Links," In Proceedings of the SPIE, Free Laser Communication Technologies, 2007, doi: 10.1117/12.698646.

[10] H. Kaushal and G. Kaddoum, "Optical Communication in Space: Challenges and Mitigation Techniques," in IEEE Communications Surveys \& Tutorials, vol. 19, no. 1, pp. 57-96, 2017, doi: 10.1109/COMST.2016.2603518.

[11] N. H. Md Noor, A. W. Naji and W. Al-Khateeb, "Performance analysis of a free space optics link with multiple transmitters/receivers," IIUM Engineering Journal, vol. 13, no. 1, pp. 49-58, 2012, doi: 10.31436/iiumej.v13i1.271.

[12] A. Niaz, F. Qamar, M. Ali, R. Farhan and M. K. Islam, "Performance analysis of chaotic FSO communication system under different weather conditions," Trans Emerging Tel Tech, no. 30, no. 2, 2018, doi: 10.1002/ett.3486

[13] J. Singh and N. Kumar, "Performance analysis of different modulation format on free space optical communication system," Optik Int. J. Light Electron. Opt., vol. 124, no. 20, pp. 4651-4654, 2013, doi: 10.1016/j.ijleo.2013.02.014.

[14] R. Kaur and S. Dewra, "Duobinary Modulation Format for Optical System-A Review," International Journal of Advanced Research in Electrical, Electronics and Instrumentation Engineering, vol.3 no, 8, pp. 11039-11046, pp. 11039-11046, 2014, doi: 10.15662/ijareeie.2014.0308012.

[15] R. Baiwa and P. Verma, "Performance Analysis of FSO System for Advanced Modulation Formats Under Different Weather Conditions," 2018 Second International Conference on Intelligent Computing and Control Systems (ICICCS), 2018, pp. 1490-1495, doi: 10.1109/ICCONS.2018.8663090.

[16] P. Sharma and H. Sarangal, "Performance Evaluation of Multiple Transceiver FSO for different Weather Conditions," International Journal of Signal Processing, Image Processing and Pattern Recognition, vol.8, no.12 pp.149-156, 2015, doi: 10.14257/ijsip.2015.8.12.15. 
[17] Z. Wang, A. Chowdhury and P. R. Prucnal, "Optical CDMA Code Wavelength Conversion Using PPLN to Improve Transmission Security," in IEEE Photonics Technology Letters, vol. 21, no. 6, pp. 383-385, March15, 2009, doi: 10.1109/LPT.2008.2011473.

[18] S. S. Muhammad, B. Flecker, E. Leitgeb and M. Gebhart, "Characterization of fog attenuation in terrestrial free space optical links," Journal of Optical Engineering, vol. 46, no. 6, pp. 066001, June 2007, doi: $10.1117 / 1.2749502$.

[19] E. Leitgeb, M. Gebhart, U. Birnbacher, W. Kogler and P. Schrotter, "High availability of hybrid wireless networks," Proc. SPIE 5465, Reliability of Optical Fiber Components, Devices, Systems, and Networks II, vol. 5654, pp 238-249, 2006, doi: 10.1117/12.545456.

[20] O. Bouchet, T. Marquis, M. Chabane, M. Alnaboulsi and H. Sizun, "FSO and quality of service software prediction," Proc. SPIE 5892, Free-Space Laser Communications V, Vol. 5892, pp.01-12, 2005, doi: $10.1117 / 12.614912$.

[21] A. Kaur and A. Kaur Brar, "A Detailed Comparison of Different Modulations in High Capacity Mode Division Multiplexed Inter-Satellite Optical Wireless Communication System," in International Journal of Engineering and Applied Sciences (IJEAS), vol. 6, no. 8, pp. 92-95, 2019, doi: 10.31873/IJEAS.6.8.2019.30.

[22] N. Badhan and N. Sharma, "Comparative analysis of CSRZ, DB and DPSK modulation formats for single channel 40Gb/s system," 2014 International Conference on Medical Imaging, m-Health and Emerging Communication Systems (MedCom), 2014, pp. 95-100, doi: 10.1109/MedCom.2014.7005983.

[23] P. Kaur, A. Gupta and M. Chaudhary, "Comparative analysis of Inter satellite Optical Wireless Channel for NRZ and RZ modulation formats for different levels of input power," Procidia Computer Science, vol 58, pp. 572-577, 2015, doi: 10.1016/j.procs.2015.08.075.

[24] H. Kaur Gill, N. S. Grewal and G. K. Walia, "Comparative investigation of CSRZ-DQPSK, DRZ-DQPSK, and MDRZ-DQPSK modulation techniques in MDM IS-OWC system," Microwave and Optical Technology , vol. 61, no. 7, pp. 1802-1809, Jul 2019, doi: 10.1002/mop.31807.

[25] P Kaur and B Utreja, "Performance Analysis of Advanced Modulation Formats in Inter-Satellite Optical Wireless Communication System," International Journal of Computer Science Engineering and Technology (IJCSET), vol. 5, no. 6, pp. 146-148, 2015

[26] H. Kaur and H. Kaur, "Study of Advanced Intensity and Phase Modulation Formats for Is-OWC DWDM System," International Journal of Engineering Technology, Management and Applied Sciences, Vol. 5, 2017.

\section{BIOGRAPHIES OF AUTHORS}
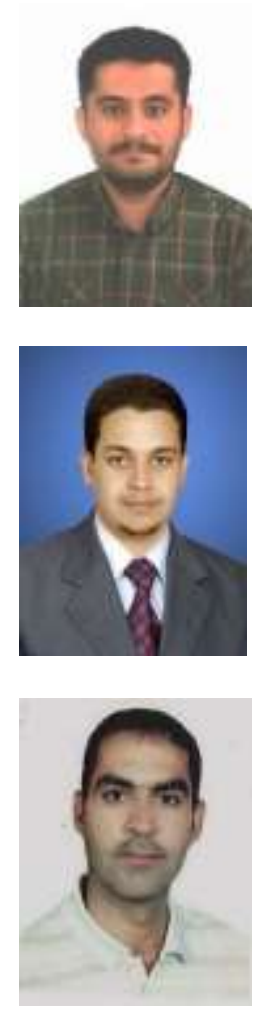

Mustafa H. Ali was born in Baghdad, Iraq, in 1990. He received the B.S. degree from Middle Technical University (MTU), Baghdad, Iraq in 2012, in specialty Computer Technical Engineering and the M.S. degree from Kazan State Technical University (named after A. N. Tupolev) Kazan, Russia in 2016, In specialty Info-communication technology and communication systems. He is currently working as a lecturer at Department of Basic Sciences/College of Dentistry/Mustansiriyah University. His research interests include Optical Communication Engineering, Passive Optical Network, Radio Over Fiber and Optical Wireless Communications.

Hazim M. Ali Issa was born in Al-Najaf Al-Ashraf, Iraq, in 1982. He received the B.S. degree from Mustansiriyah University, Baghdad, Iraq, in 2006, In specialty Computer Sciences. Also, he received the M.S. degree from Engineering Colloge of Ferdowsi University in Mashhad, Iran in 2017, In specialty Artificial Intelligence and Robotics. He is currently working as a lecturer at Department of Computer Science in Ministry of Education-General Directorate of Education Al-Najaf Al-Ashraf. His research interests include Computer Softwere, Networks, Artificial Intelligence and Robotics.

Dr. Tariq A. Hassan was born in Baghdad, Iraq, in 1976. He received the B.S. degree from ALRafidain University, Baghdad, Iraq, in 2000, in specialty Computer Science and the M.S. degree from Al-Mustansiriyah University, collage of Sceince, Baghdad, Iraq, in 2005., In specialty Digital 11signal processing (DSP). Ph.D. degree is received from Southampton University in computer science, UK in 2012. He is currently working as a lecturer at Department of Computer Sciences /College of Education/Mustansiriyah University. His research interest's robust speaker identification and verification, speaker recognition, Speech compression and hiding. Signal analysis and chaotic technique application 